\title{
Editorial
}

Lifestyle

Genomics

\section{Modulation of the Epigenome by Nutrition and Xenobiotics during Early Life and across the Life Span: The Key Role of Lifestyle}

\author{
Rosita Gabbianelli \\ Unit of Molecular Biology, School of Pharmacy, University of Camerino, Camerino, Italy
}

Epigenetic modulation due to environmental factors (i.e., nutrition, xenobiotics, physical activity, stress, etc.) has a key role in cell differentiation during early life and across the life span because it can actively influence gene expression in a cell- and tissue-specific manner.

A key window of epigenetic plasticity has been observed during the first 1,000 days of life when the epigenome can program differentiation of stem cells and primordial germ cells. Maternal and neonatal nutrition can modulate the offspring's epigenome, leading to the development of a healthy/ unhealthy phenotype in adulthood [1].

DNA methylation is a key epigenetic mechanism in the control of gene expression. The methylation of $\mathrm{CpG}$ islands in a gene's promoter region has been associated to a progressive switching off of the gene, and the availability of methyl groups required for methylation depends on a folate-rich diet (e.g., spinach, green leaves, peas, asparagus, etc.) as well as sufficient levels of B12 and B6 vitamins $[2,3]$. Deficiencies in these micronutrients during early life have been associated with increased risk of cardiovascular, metabolic, and neurological diseases later in life [4, 5]. In addition to nutrients, other lifestyle factors such as smoking, alcohol, stress, and food pesticides have also been reported to increase the risk of disease development

\section{KARGER}

(c) 2018 S. Karger AG, Basel

E-Mail karger@karger.com

www.karger.com/lfg in adulthood according to genetic variations [5]. For example, alcohol can interfere with methyl donor transfer from folate to methyl tetrahydrofolate, thus perturbing the one-carbon metabolism (folate) pathway [6]. A maternal deficiency in folate has been associated with decreased availability in methyl groups. Furthermore, alcohol consumption during pregnancy can perturb normal neuronal development and promote proinflammatory cytokine release in offspring $[5,6]$. Similarly, a maternal high-fat diet can contribute to proinflammatory cytokine production, and maternal obesity can promote changes in methylation patterns [4]. Finally, exposure to pesticides and metals during pregnancy and/or early life can promote inflammation, neurodevelopmental disorders, and progressive neurodegeneration later in life $[7,8]$.

Notably, epigenetics is an active regulatory mechanism throughout a person's entire life. For example, a diet that is low in folate, B6, and B12 during adulthood can reduce the level of available methyl groups for adenosylmethionine, which is used by DNA methyltransferases to methylate $\mathrm{CpG}$ islands. In cancer, the epigenome is changed significantly. Aberrant (promoter) hypermethylation and genome-wide hypomethylation have been reported [9]. Hypermethylation of CpG-enriched regions 


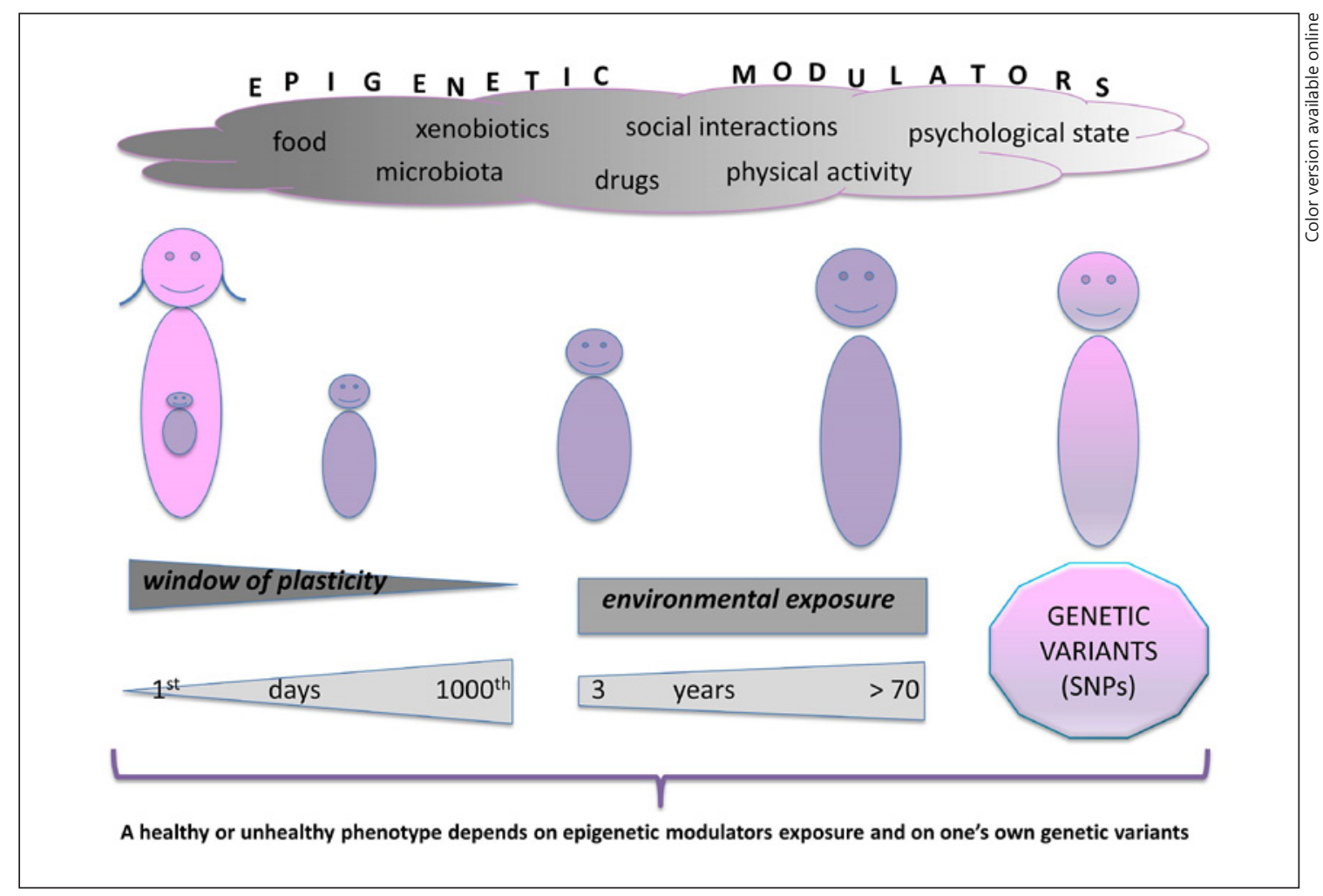

Fig. 1. Epigenetic modulators across the life span and genetic variability can modulate the individual responses towards a healthy or unhealthy status.

associated with genes involved in tumor suppression and DNA repair leads to their inactivation, while global hypomethylation has been associated with the induction of genes responsible for oncogene activation and chromosome instability [9].

Histone modification is the second most important epigenetic mechanism involved in chromatin remodelling. Functional groups (e.g., acetyl, methyl, and phosphate) obtained by the catabolic pathways of macromolecules, can shift heterochromatin-silencing of genes to maintain normal gene function.

In this context, the diet has an important role in the modulation of gene expression, because it provides the key macro- and micronutrients required for the production of functional groups and substrates able to modulate the epigenome. Important in this context is the role of mitochondria, the major center of metabolism, where acetyl groups are produced following $\beta$-oxidation of fatty acids or the decarboxylation of pyruvate, and where amino acid metabolism can provide intermediates of the citric acid cycle that modulate the methionine cycle. For ex- ample, acetyl groups can mask the positive charge of lysine residues, leading to chromatin decondensation, which is a prerequisite for the onset of gene expression. Recently, much interest has been dedicated to the investigation of the correlation between mitochondrial dysfunction and its possible influence on the nuclear epigenome [10]. Attention has been focused on the level of reduced nicotinamide adenine dinucleotide (NADH) in mitochondria, suggesting a possible connection with methionine metabolism and the regulation of nuclear DNA methylation [11].

Foods (e.g., vegetables, legumes, fruits, cereals, tea, and soy) that contain bioactive components (e.g., polyphenols, quercetin, sulforaphane curcumin, genistein, etc.) can modulate gene expression and the epigenome. Thus, studies that aim to understand the molecular mechanisms associated with nutrient-gene interactions and nutrient-epigenome modulation represent a primary area of investigation in the field of nutrigenomics.

Particular attention should be given to the quality of food consumed, because xenobiotic (e.g., pesticides and
10

Lifestyle Genomics 2018;11:9-12 DOI: $10.1159 / 000490751$
Gabbianelli et al. 
metals) residues that are used for pest control are detected in foods [12]. Studies using animal models, as well as humans working on farms, show increased levels of pesticide metabolites in urine [5]. These chemicals can have an impact on gene expression and epigenetic mechanisms $[13,14]$. For example, pesticide residues in foods have been associated with an increased risk for the development of neuronal damage $[5,15]$ and obesity $[16]$ according to individual genetic variations and the epigenetic modifications that may be induced and potentially inherited in the unexposed offspring $[5,17,18]$. In this regard, promoting the consumption of organic foods may be beneficial particularly during the sensitive window of epigenetic plasticity (i.e., early life) to prevent the risk of developing neuronal damage $[5,8,14]$.

It is also noteworthy that the consumption of vegetables and fruits provides complex carbohydrates (i.e., fiber) that enable gut bacteria to produce short-chain fatty acids, which have an active role in the regulation of cytokine production. In particular, proinflammatory cytokines (e.g., TNF- $\alpha$, IL-1 $\beta$, IL-6, etc.) can increase with aging [19], and short-chain fatty acids produced by the microbiota are able to control their production and can promote the release of protective IL-10 [5]. In contrast, a diet with high meat, choline, carnitine, and fat intake in some individuals can promote trimethylamine- $\mathrm{N}$-oxide formation, which mediates inflammation. The control of inflammation and oxidative stress represents a key strategy in the maintenance of health [20], and lifestyle (e.g., diet, environmental xenobiotics, physical activity, stress, social interactions, etc.) has a key role in the maintenance of cellular homeostasis.

However, the epigenome is not the sole determinant of a person's health. Indeed, the underlying genetic makeup of an individual also plays a key role in determining how a person responds to a particular nutritional/environmental stimulus. A future goal for optimizing a person's health will depend on personalized nutrition [21, 22]. Metabolic responses to food depend on an individual's genetic make-up and the 10 million single-nucleotide polymorphisms that characterize the human genome that are unique to each and every individual. Nutrigenetics aims to provide explanations regarding the complex scenario of how genetic variants influence responses to diet. As such, assessing different responses to foods represents the hard and ambitious goal for personalized nutrition.

In conclusion, epigenetics and genetics are key players in the determination of health. Since genetics cannot be modified, continued research will improve the knowledge of gene-nutrient interactions to generate useful in- formation for personalized nutritional recommendations. In contrast, epigenetics will prove more informative regarding the specific relationships between the quality and quantity of bioactive food compounds that are able to modulate gene expression by regulating DNA methylation and histone modification. What we have to consider now is that the choices a person makes during their entire life can have a significant impact on their health by regulating gene expression and the epigenome (Fig. 1). It is therefore crucial to educate future generations on these important topics and how they can influence a person's quality of life.

The goal of the 3rd European Summer School on Nutrigenomics is to give new insights on 5 key topics: the first is the key role of early-life nutrition in the programming of adult health. The second will examine new aspects related to the impact of nutrients on gene expression and the epigenome in adulthood and the individual response to diet. Third, the importance of organic foods will be discussed to investigate the main role of food xenobiotics in the modulation of gene expression. Fourth, insights into how lifestyle factors (diet, physical activity, sleep, leisure-time, and sedentary behavior) modify gene expression will be investigated. The final topic will describe nutrigenomic biomarkers in blood, urine, and saliva that are useful to screen for epigenetic changes to monitor human health. The abstracts associated with the 3rd European Summer School on Nutrigenomics presented with this Editorial highlight the breadth of research addressed by attendees.

References

Lifestyle Genomics 2018;11:9-12

1 Hanley B, Dijane J, Fewtrell M, Grynberg A, Hummel S, Junien C, Koletzko B, Lewis S, Renz H, Symonds M, Gros M, Harthoorn L, Mace K, Samuels F, van Der Beek EM: Metabolic imprinting, programming and epigenetics - a review of present priorities and future opportunities. Br J Nutr 2010; 104(S1):S1-S25.

2 Naninck EF, Oosterink JE, Yam KY, de Vries LP, Schierbeek H, van Goudoever JB, VerkaikSchakel RN, Plantinga JA, Plosch T, Lucassen PJ, Korosi A: Early micronutrient supplementation protects against early stress-induced cognitive impairments. FASEB J 2017;31: 505-518.

3 McGee M, Bainbridge S, Fontaine-Bisson B: A crucial role for maternal dietary methyl donor intake in epigenetic programming and fetal growth outcomes. Nutr Rev 2018;76:469-478.

4 Menendez-Castro C, Rascher W, Hartner A: Intrauterine growth restriction - impact on cardiovascular diseases later in life. Mol Cell Pediatr 2018;5:4. 
5 Gabbianelli R, Damiani E: Epigenetics and neurodegeneration: role of early-life nutrition. J Nutr Biochem 2018;57:1-13.

6 Nakhoul MR, Seif KE, Haddad N HG: Fetal alcohol exposure: the common toll. J Alcohol Drug Depend 2017;5:257.

7 Modgil S, Lahiri DK, Sharma VL, Anand A: Role of early life exposure and environment on neurodegeneration: implications on brain disorders. Transl Neurodegener 2014;3:9.

8 Carloni M, Nasuti C, Fedeli D, Montani M, Amici A, Vadhana MSD, et al: The impact of early life permethrin exposure on development of neurodegeneration in adulthood. Exp Gerontol 2012;47:60-66.

9 Gerhauser C: Cancer chemoprevention and nutri-epigenetics: state of the art and future challenges. Top Curr Chem 2013;329:73-132.

10 Van der Wijst MG, Van Tilburg AY, Ruiters MH, Rots MG: Experimental mitochondriatargeted DNA methylation identifies $\mathrm{GpC}$ methylation, not $\mathrm{CpG}$ methylation, as potential regulator of mitochondrial gene expression. Sci Rep 2017;7:177.

11 Lozoya OA, Martinez-Reyes I, Wang $\mathrm{T}$, Grenet D, Bushel P, Li J, Chandel N, Woychik RP, Santos JH: Mitochondrial nicotinamide adenine dinucleotide reduced (NADH) oxidation links the tricarboxylic acid (TCA) cycle with methionine metabolism and nuclear DNA methylation. PLoS Biol 2018; 16:e200570.

12 Karmaus AL, Trautman TD, Krishan M, Filer DL, Fix LA: Curation of food-relevant chemicals in ToxCast. Food Chem Toxicol 2017; 103:174-182.
13 Skinner MK: Endocrine disruptors in 2015: epigenetic transgenerational inheritance. Nat Rev Endocrinol 2016;12:68-70.

14 Collotta M, Bertazzi PA, Bollati V: Epigenetics and pesticides. Toxicology 2013;307: 35-41.

15 Nasuti C, Fattoretti P, Carloni M, Fedeli D, Ubaldi M, Ciccocioppo R, Gabbianelli R: Neonatal exposure to permethrin pesticide causes lifelong fear and spatial learning deficits and alters hippocampal morphology of synapses. J Neurodev Disord 2014;6:7.

16 Alonso-Magdalena P, Rivera FJ, GuerreroBosagna C: Bisphenol-A and metabolic diseases: epigenetic, developmental and transgenerational basis. Environ Epigenet 2016; 2:dvw022.

17 Bordoni L, Nasuti C, Mirto M, Caradonna F, Gabbianelli R: Intergenerational effect of early life exposure to permethrin: changes in global DNA methylation and in Nurr1 gene expression. Toxics 2015;3:451-461.

18 Manikkam M, Haque MM, Guerrero-Bosagna C, Nilsson E, Skinner M: Pesticide methoxychlor promotes the epigenetic transgenerational inheritance of adult onset disease through the female germline. PLoS One 2014; 9:e102091.

19 Van Der Lugt B, Rusli F, Lute C, Lamprakis A, Salazar E, Boekschoten MV, Hooiveld GJ, Müller M, Vervoort J, Kersten S, Belzer C, Kok DEG, Steegenga WT: Integrative analysis of gut microbiota composition, host colonic gene expression and intraluminal metabolites in aging C57BL/6J mice. Aging (Albany NY) 2018;10:930-950
20 Dossus L, Franceschi S, Biessy C, Navionis AS, Travis RC, Weiderpass E, Scalbert A, Romieu I, Tjønneland A, Olsen A, Overvad K, Boutron-Ruault MC, Bonnet F, Fournier A, Fortner RT, Kaaks R, Aleksandrova K, Trichopoulou A, La Vecchia C, Peppa E, Tumino R, Panico S, Palli D, Agnoli C, Vineis P, Bueno-de-Mesquita HBA, Peeters PH, Skeie G, Zamora-Ros R, Chirlaque MD, Ardanaz E, Sánchez MJ, Ramón Quirós J, Dorronsoro M, Sandström M, Nilsson LM, Schmidt JA, Khaw KT, Tsilidis KK, Aune D, Riboli E, Rinaldi S: Adipokines and inflammation markers and risk of differentiated thyroid carcinoma: The EPIC study. Int J Cancer 2018;142:13321342.

21 De Roos B, Brennan L: Personalised interventions - a precision approach for the next generation of dietary intervention studies. Nutrients 2017 9;E847.

22 Ramos-Lopez O, Milagro FI, Allayee $\mathrm{H}$, Chmurzynska A, Choi MS, Curi R, De Caterina R, Ferguson LR, Goni L, Kang JX, Kohlmeier M, Marti A, Moreno LA, Pérusse L, Prasad C, Qi L, Reifen R, Riezu-Boj JI, SanCristobal R, Santos JL, Martínez JA: Guide for current nutrigenetic, nutrigenomic, and nutriepigenetic approaches for precision nutrition involving the prevention and management of chronic diseases associated with obesity. J Nutrigenet Nutrigenomics 2017;10: 43-62. 